\title{
Familial nonrandom inactivation linked to the $X$ inactivation centre in heterozygotes manifesting haemophilia A
}

\author{
Maria Patrizia Bicocchi ${ }^{1}$, Barbara R Migeon ${ }^{2}$, Mirella Pasino*, ${ }^{*}$, Tiziana Lanza ${ }^{1}$, \\ Federico Bottini $^{1}$, Elio Boeri ${ }^{1}$, Angelo C Molinari ${ }^{1}$, Fabio Corsolini ${ }^{3}$, Cristina Morerio ${ }^{4}$ \\ and Maura Acquila ${ }^{1}$
}

\begin{abstract}
${ }^{1}$ Thrombosis and Haemostasis Unit, Department of Haematology and Oncology, Giannina Gaslini Institute, Genova, Italy; ${ }^{2}$ McKusick Nathans Institute of Genetic Medicine, The Johns Hopkins University, Baltimore, MD, USA;

${ }^{3}$ Laboratory of Pre- and Post-natal Diagnosis of Metabolic Diseases, Giannina Gaslini Institute, Genova, Italy; ${ }^{4}$ Cancer Cytogenetics Unit, Giannina Gaslini Institute, Genova, Italy
\end{abstract}

A basic tenet of the Lyon hypothesis is that $\mathrm{X}$ inactivation occurs randomly with respect to parental origin of the $X$ chromosome. Yet, nonrandom patterns of $X$ inactivation are common - often ascertained in women who manifest recessive $X$-linked disorders despite being heterozygous for the mutation. Usually, the cause of skewing is cell selection disfavouring one of the cell lineages created by random $X$ inactivation. We have identified a three generation kindred, with three females who have haemophilia A because of extreme skewing of $X$ inactivation. Although they have both normal and mutant factor VIII (FVIII) alleles, only the mutant one is transcribed; and, they share an XIST allele that is never transcribed. The skewing in this case seems to result from an abnormality in the initial choice process, which prevents the chromosome bearing the mutant FVIII allele from being an inactive $\mathrm{X}$.

European Journal of Human Genetics (2005) 13, 635-640. doi:10.1038/sj.ejhg.5201386

Published online 2 March 2005

Keywords: haemophilia A; XIST; nonrandom X inactivation; haemophiliac females

\section{Introduction}

Haemophilia A (HA) is a recessive disorder caused by a mutation of the factor VIII $(F 8 C)$ gene on the distal long arm of the X chromosome, in Xq28. Usually only males are affected, while heterozygous carriers are asymptomatic. The extremely rare female cases with HA phenotype have been ascribed to mutations in both $F 8 C$ alleles ${ }^{1,2}$ or a second mutation affecting the von Willebrand factor gene, also in the clotting pathway. ${ }^{3}$ There have been several reports of females who manifest HA due to skewed $\mathrm{X}$

*Correspondence: $\operatorname{Dr}$ M Pasino, Thrombosis and Haemostasis Unit, Department of Haematology and Oncology, G Gaslini Institute, Largo G Gaslini no. 5, Genova 16147, Italy. Tel: + 390105636277 .

Fax: + 39010 386204; E-mail: mirellapasino@ospedale-gaslini.ge.it Received 22 July 2004; revised 12 January 2005; accepted 19 January 2005 inactivation. Some have cytogenetic abnormalities such as $\mathrm{X} /$ autosome translocations. ${ }^{4}$ Some are monozyotic twins ${ }^{5}$ who are often discordant for a variety of X-linked disorders because of an unexplained disturbance in the randomness of $\mathrm{X}$ inactivation in twins. ${ }^{6}$ Others are isolated cases of females who are heterozygous for an $F 8 C$ mutation, but whose mutant gene is expressed in more than the $50 \%$ of cells expected based on random $\mathrm{X}$ inactivation. ${ }^{7-9}$ The cause of skewed $\mathrm{X}$ inactivation is sometimes attributable to a selective advantage of a coincident, but unrelated mutation, like the woman heterozygous not only for HA but also for incontinentia pigmenti - a disease known to adversely affect cell proliferation. ${ }^{10}$ However, in most cases, the cause of the skewing is unknown.

The inactivation of an $\mathrm{X}$ chromosome occurs early in development, is maintained in a clonal manner through- 
out subsequent cell divisions and, by this process, the dosage of proteins encoded by genes on the $\mathrm{X}$ chromosome is equalised in males and females. The choice of which of the two $\mathrm{X}$ chromosomes is the active one is usually random. Although stochastic events may account for a less than equal distribution of the two cell lineages in the mosaic female (as the result of the limited number of founder cells in specific tissues), excessively skewed $\mathrm{X}$ inactivation (>95\%) is rarely found in normal females. ${ }^{11}$

Nonrandom inactivation can arise either from a bias in the original decision as to which chromosome is the active one or from subsequent selective pressures, restricted to a specific cell lineage as observed for some X-linked lymphoproliferative disorders. ${ }^{12}$ On the one hand, the most common cause of skewing of $\mathrm{X}$ inactivation are mutations that convey a proliferative advantage or disadvantage to a cell. ${ }^{13}$ The fact that human females are heterozygous at so many X-linked loci sets up a growth competition between the cell lineages, and one lineage can outgrow the other. Women, heterozygous for mutations causing Lesch-Nyhan syndrome or adrenoleukodystrophy, often have extremely skewed inactivation patterns because of cell selection. Skewed $\mathrm{X}$ inactivation of this kind has been shown to be a common feature of X-linked mental retardation disorders. ${ }^{14}$ On the other hand, if the bias is caused by mutations affecting the initial decision, then it is likely to affect the X inactivation centre (XIC in human, $\mathrm{Xic}$ in mouse). This is the region of the $\mathrm{X}$ chromosome that includes the $\mathrm{X}$ inactive transcript gene, XIST, the key gene in the cis inactivation pathway, located in the proximal long arm, in Xq13.2. The noncoding RNA transcribed only from the XIST locus on an inactive $\mathrm{X}$ surrounds the chromosome and induces the chromatin modifications and transcriptional silence characteristic of an inactive $\mathrm{X}$. The centre also includes other DNA sequences that are believed to have a regulatory role in the process at least in the mouse. ${ }^{15}$ And it is likely that some autosomal genes interact with the Xic. ${ }^{16}$ The human XIST gene has been fully sequenced ${ }^{17,18}$ but its functional domains have not yet been fully identified. Yet, it is clear that an X chromosome cannot be inactive without a functional XIST gene. Deletions of the locus completely prevent cis inactivation. It is likely that mutations, which decrease its activity, will interfere with the chromosome's ability to inactivate. As only the X chromosome with a normal XIST locus can inactivate, XIST mutations would lead to nonrandom $\mathrm{X}$ inactivation. In addition, so that one $\mathrm{X}$ remains active in each diploid cell, one XIST locus per cell must be repressed; hence, some element(s) in the XIC is needed to receive the signal-mediating XIST repression. Mutations that interfere with response to that signal, or enhance the probability that XIST will be repressed, might also lead to nonrandom inactivation.

Yet, it has been difficult to identify such mutations among individuals with skewed $\mathrm{X}$ inactivation or to show that the skewing is primary, attributable to the process of cis inactivation. As most of the cases have been sporadic, the families are not large enough to show cosegregation of the skewing with the $\mathrm{X}$ inactivation centre. Here, we describe a family in which three females, in two generations, affected with HA show extreme skewing of $\mathrm{X}$ inactivation that seems to segregate with a specific XIST allele.

\section{Case report}

The proband (Figure 1, III-4), an 8-year-old boy, was admitted to the hospital in 1996 because of bleeding, swelling and reduced osteoarticular mobility of his right elbow. Laboratory studies showed that he was affected with $\mathrm{HA}$, as the residual activity of factor VIII:C was $6 \%$ of normal. FVIII binding capacity by the von Willebrand factor and VWF:Ag assays were normal. During the next 5 years, he had two other spontaneous elbow bleeds and one severe haemarthrosis of the ankle. When needed, treatment with FVIII recombinant concentrate (rhFVIII) always resolved the bleeding.

The family history revealed that bleeding disorders affected three female members of the family, his mother, his maternal aunt and her daughter (Figure 1). The mother (II-4) and the aunt (II-2), born in 1952 and 1949, respectively, had the typical clinical picture of HA. Their 6\% FVIII:C residual activity was compatible with a diagnosis of HA. Abnormal bleeding occurred following surgical interventions, such as tooth extraction (II-4) or tonsillectomy (II-2), and was controlled with plasma, blood concentrate and, more recently, rhFVIII. Prophylaxis with rhFVIII was used during their obstetrical care.

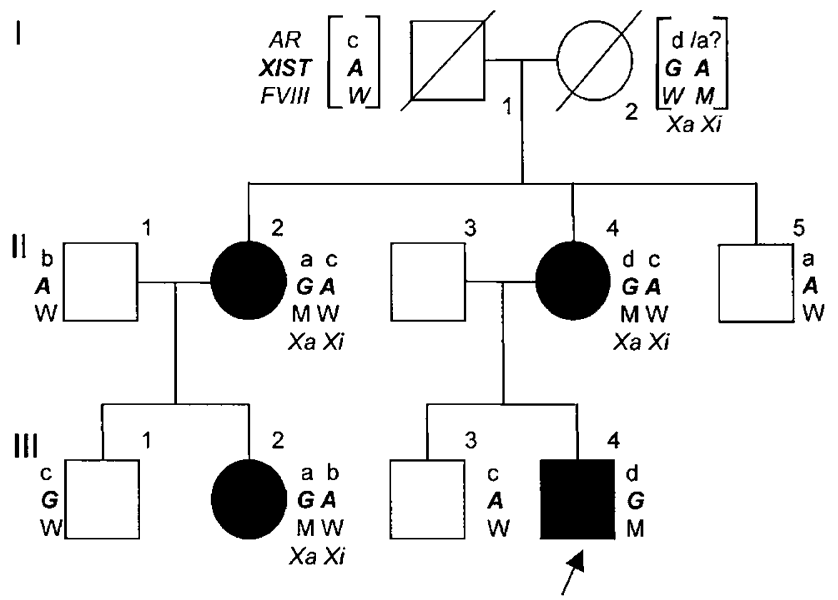

Figure 1 Pedigree, showing affected proband (black square, arrow) and heterozygotes (black circles), and segregation of the relevant haplotypes. $A R$ alleles: (a, b, $c$ and d); XIST alleles: $A, G$; F8C alleles: mutant $(\mathrm{M})$ and normal $(\mathrm{W})$; active $(\mathrm{Xa})$ and inactive $(\mathrm{Xi}) \mathrm{X}$ chromosomes; [ ]: the alleles deduced from the transmission pattern assuming that the Gll females have the same healthy father. 
The female cousin of the proband (III-2), born in 1985, had an episode of dental bleeding, controlled with rhFVIII. All the females manifesting haemophilia had no other clinical abnormalities.

\section{Methods}

\section{Cytogenetic studies}

Cytogenetic analyses were performed by standard methods on PHA-stimulated peripheral blood lymphocytes using high-resolution banded chromosome. A total of 100 metaphases was examined for each patient sample.

\section{Molecular studies}

Genomic DNA analysis Citrated blood samples were obtained following informed consent from individuals from the second and third generation in this kindred (Figure 1). Skin fibroblasts from III-2 and uroepithelial and buccal mucosa cells from II-4 were also studied. Genomic DNA was extracted from leucocytes and fibroblasts by standard procedures, and from uroepithelial and buccal cells by boiling.

RNA analysis Complementary DNA (cDNA) was made from peripheral blood RNA using reverse transcriptase (RNA synthesis kit, Clontech, Palo Alto, CA, USA).

\section{Mutation identification}

F8C The entire F8C gene coding region, including flanking splicing sites, was amplified using appropriate primers. ${ }^{19}$ The promoter sequence and the polyadenylation site were analysed using the primers described elsewhere. ${ }^{20}$ Equal volumes of PCR product from each sample patient and from a wild-type control male were mixed and treated to obtain heteroduplexes. Screening of the mutation was performed using DHPLC (DHPLC Helix ${ }^{\mathrm{TM}}$ Varian system - Varian Analytical Instruments, CA, USA). Any sample showing an abnormal peak pattern was sequenced using a fluorescent ABI PRISM BigDye terminator kit (Applied-Biosystem, UK), and the mutation was characterised. The mutation was confirmed on a second, independent, amplified PCR sample. The diagnosis of carrier status in the haemophiliac's mother and in related females was completed by searching for the mutation. All mutations identified were confirmed by sequencing.

XIST PCR primers were used to amplify the minimal promoter of XIST in all the affected members of the family as described, ${ }^{21}$ and the PCR product was sequenced directly.

\section{$\mathrm{X}$ chromosome inactivation analysis}

The pattern of $\mathrm{X}$ inactivation was determined using the HUMARA assay of Allen et al. ${ }^{22}$ Using digestion with a methylation-sensitive endonuclease, coupled with PCR analysis of the CAG polymorphic repeat in the first exon of the human androgen receptor $(A R)$ gene, we could distinguish active from inactive $X$, and determine the proportion of the cells with a given active X. Briefly, genomic DNA was incubated overnight in restriction buffer with HpaII ( $8 \mathrm{U} / \mu \mathrm{g}$ DNA). Amplified products of the $A R$ gene were obtained from both digested and undigested samples of females, and subjected to $9 \%$ nondenaturing polyacrylamide gel electrophoresis. Allele bands were detected on silver-stained gel. In male family members, the HUMARA haplotype was determined on PCR products from undigested DNA.

$\mathrm{X}$ inactivation was also analysed by determining which of the XIST alleles was transcribed using the single-base polymorphism G15944A to distinguish XIST alleles. ${ }^{23}$

\section{Results}

The proband and his mother had a normal karyotype. Sequencing of exon 8 of the $F 8 C$ gene in the proband revealed a $C$ to $G$ transversion, which introduces an arginine to glycine substitution at amino acid codon 372 . His mother and the other affected females had the same mutation, which is described in the FVIII mutation Database (HAMSTeRS; http://europium.csc.mrc.ac.uk ${ }^{24}$ ) in the same nucleotide but with a different amino acid substitution.

The results of the $\mathrm{X}$ inactivation studies demonstrated that both the proband's mother and his aunt were heterozygous at the HUMARA locus (Figure 1; Figure 2, lanes 1 and 3); they had two different maternally inherited alleles as the result of a meiotic crossover that had occurred between the HUMARA and the F8C genes. In addition, the pattern of $\mathrm{X}$ inactivation was completely skewed in all three affected females. Figure 2 compares the PCR products amplified before, (lanes 1, 3 and 5) and after HpaII digestion (lanes 2, 4 and 6), respectively. The inactive chromosome $\mathrm{X}$ was always of paternal origin in affected females. This extreme skewing (>95\%) was present not only in their leucocytes but also in the skin fibroblasts, uroepithelium and buccal mucosa - all the tissues analysed

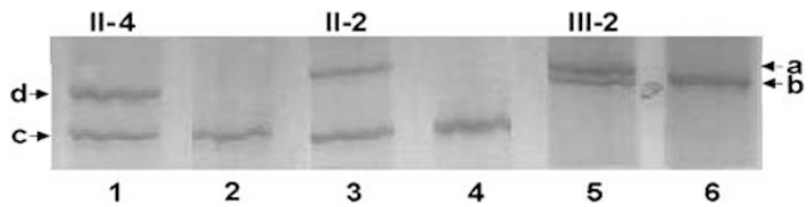

Figure $2 \mathrm{X}$ inactivation patterns by the HUMARA assay show complete skewing in heterozygotes II-4 (lanes 1 and 2), II-2 (lanes 3 and 4) and III-2 (lanes 5 and 6). AR alleles: ( $a, b, c$ and d). PCR samples amplified for $A R$ before (lanes 1, 3 and 5) and after (lanes 2, 4 and 6) digestion with Hpall run on a $9 \%$ nondenaturing acrylamide silverstained gel. Note: the $b$ and $c$ alleles are undigested and therefore on the inactive $X$. 


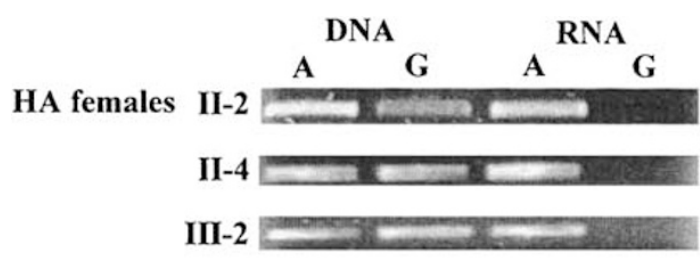

HA male III-4 2ans

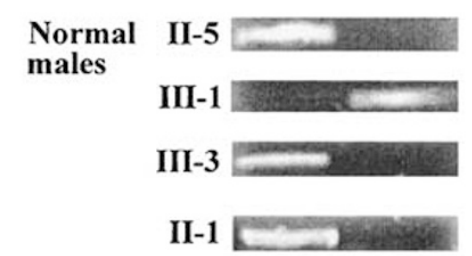

Control female

Figure 3 Analysis of the expressed XIST $15944 G \rightarrow$ A polymorphism shows skewing in the heterozygotes, with the XIST $G$ allele (unexpressed) consistently on their active X. XIST alleles (DNA: $A, G$ ) were determined from genomic DNA. The inactive $\mathrm{X}$ allele (RNA: $A, G$ ) was determined from cDNA. Confirming findings from the HUMARA assay, only one allele was expressed in all of them, and that was the XIST $A$ allele (always on the inactive X). A normal XIST A/G heterozygous female is shown.

from these heterozygotes (data not shown). Moreover, unbalanced inactivation was also supported by RT-PCR of F8C exon 8 from an affected female sample: direct sequencing of the amplified fragment demonstrated that the only allele expressed was the mutated one (data not shown).

All three affected females were also heterozygous for the G15944A XIST polymorphism (Figures 1 and 3). This enabled us to independently confirm the complete skewing of $\mathrm{X}$ inactivation observed by the HUMARA methylation assay: only the XIST $A$ allele, of paternal origin, was expressed from their inactive $\mathrm{X}$, so that the maternal XIST $G$ allele had to be on the active X chromosome in all their cells (Figure 3). The G containing XIST allele was also seen in the proband's normal male cousin (III-1), so that a crossover had occurred between the F8C and XIST alleles.

The sequence analysis of the XIST minimal promoter did not reveal any abnormalities of the amplified fragment (data not shown).

\section{Discussion}

The family that we have studied is segregating a missense mutation at codon 372 of the F8C gene that changes an amino acid in the thrombin activation site. The unusual feature of this family is that there are three females affected with classic HA, and all of them are heterozygous for this mutation.
Usually, the presence of a wild-type $F 8 C$ gene protects the female from manifesting a bleeding disorder. If $\mathrm{X}$ inactivation is random with respect to the parental origin of the $\mathrm{X}$ - which most often is the case - then there will be sufficient cells expressing the wild-type gene to prevent abnormal bleeding in the female. In rare cases, heterozygous females will manifest the mutation because not enough of their cells are synthesising the normal clotting factor. This can happen in females who have a single $\mathrm{X}$ chromosome, or a translocation that inactivates the normal F8C gene. However, chromosome studies in our family show that affected females have a normal karyotype.

The clinical manifestation of HA, in three females from two consecutive generations, is caused by the concomitant occurrence of two events: (i) the F8C mutation on the maternal $\mathrm{X}$ chromosome and (ii) the skewed pattern of $\mathrm{X}$ inactivation that prevents the expression of the wild-type F8C gene on the paternal X chromosomes. Although they could not be studied, both grandparents were reported to have been healthy. It is improbable that the haemophilic mutation was present in the grandfather. HA in males is usually fully penetrant, and males who do not manifest an F8C mutation on their only $\mathrm{X}$ chromosome have not been observed. The possibility of a new sperm mutation is also unlikely because both daughters have the F8C mutation. Most likely, the HA mutation in this family was transmitted on one of the grandmother's X chromosomes. The fact that she does not manifest the disorder is not surprising, as she is heterozygous; in the absence of $\mathrm{X}$ inactivation skewed to favour her mutant allele, she would not be expected to have HA.

However, $\mathrm{X}$ inactivation is skewed in her daughters and granddaughter, based on the expression of a single allele at both the HUMARA and XIST loci. It is unlikely that random stochastic events account for the skewed inactivation patterns in three females from two generations and in four different tissues.

What is the basis for the skewed inactivation in this family? The extreme nonrandom inactivation ratios that we observed could be due to cell selection, disfavouring cells with a mutation that influences the ability to proliferate. In this case, skewing of $\mathrm{X}$ inactivation patterns would have occurred after random inactivation. Although $\mathrm{HA}$ is detrimental to an individual, it does not seem to influence cell proliferation and, in fact, there is no evidence to suggest it could be advantageous to cells, as it is in this family. In addition, there is no other known disease mutation segregating in this family to predispose them to unbalanced $X$ inactivation. Could there be some other allele on the paternal $X$ that could disfavour cells in which that chromosome is the active $\mathrm{X}$ ? As females from two generations are affected and each has a different paternal X, it is unlikely that this is the case. More likely, there is some locus on the maternal $\mathrm{X}$ chromosome that is 
common to all three affected females, and this locus is responsible for its always being an active $\mathrm{X}$. The effect of such a locus might be to influence inactivation status of the chromosome at the time when the active $\mathrm{X}$ is chosen.

Two unrelated families segregating skewed $X$ inactivation were reported. ${ }^{21}$ The skewed patterns of $\mathrm{X}$ inactivation in some females were associated with a mutation in the XIST minimal promoter. The authors proposed that this mutation in the XIST minimal promoter results in preferential choice of this chromosome as the inactive $\mathrm{X}$. Notable is the fact that the extent of skewing was variable in these families; not all the females were skewed to the same extent, and most of them did not show the extreme skewing we have observed. Plenge et al ${ }^{21}$ suggested that the promoter mutation observed in their two unrelated families might be the cause of nonrandom inactivation in other families as well.

To our knowledge, ours is the first family where it is possible to demonstrate that extreme skewing has been transmitted to three females in two generations, with the same X chromosome being the active one in all of them. A likely possibility is that the $\mathrm{X}$ chromosome that is consistently active in each of these females has some abnormality within its XIC. To determine if this was true, we examined the promoter of the XIST gene in our family. However, neither the previously reported mutation nor any other mutation within the minimal promoter was found. Therefore, we can exclude that the maternal $\mathrm{X}$ chromosome carries a mutation in the minimal promoter that blocks the expression of its own XIST. Of interest, Pereira and $Z^{2} z^{25}$ also did not find the C43G promoter mutation in 66 females with highly skewed $\mathrm{X}$ inactivation, so it seems not to be a common cause of skewing.

Yet, XIST, or other regulatory sequences in the XIC, remain the best candidates for the locus responsible for the extreme skewing that we observed. The analysis of the G15994A polymorphism within the XIST gene ${ }^{23}$ shows that each female has the same XIST allele - the one with $X I S T G$; hence, they are likely to have inherited the same XIC region. Although we know, based on transmission data, that the grandmother of the proband (I-2) is heterozygous for the XIST, having both $A$ and $G$ alleles, we cannot determine the phase with respect to her other Xlinked markers. As she had the XIST $G$ allele, it is more likely that she also had skewed X inactivation; in this case, her active X would always be the one with the XIST $G$ allele. As she did not manifest HA, her mutant F8C gene had to be coupled with the XIST A allele on her inactive X. As a consequence, the HA mutation was silent because in this case the skewing favoured cells expressing the wildtype $F 8 C$ allele. However, we cannot rule out a germinal mosaicism either for the HA mutation or for a XIC deregulation. Recombination between F8C and XIST alleles is expected to occur frequently because of the great distance between the two loci. Finding the $G$ containing
XIST allele on the active $\mathrm{X}$ in normal male, III-1 is consistent with this expectation. Therefore, based on all our observations, it seems reasonable to postulate that some kind of alteration occurred within the XIC of the maternal $X$ chromosome that prevents it from being the inactive $\mathrm{X}$, or alternatively makes it more likely to be the active $X$. However, we cannot rule out the influence of a yet unidentified, X-linked control locus or a growth advantage unknown locus. ${ }^{26,27}$

As the nonrandom $\mathrm{X}$ inactivation involves the same chromosome in all manifesting heterozygotes, and that chromosome carries the XIST G allele, it is tempting to speculate that the daughters of III-1 will have skewed $X$ inactivation in the absence of HA. As this healthy male has the XIST $G$ allele and, therefore, the same XIC region shared with the haemophiliac females, he will transmit an $\mathrm{X}$ chromosome that also is unable to inactivate. In any case, we believe that the best interpretation of our studies is that the haemophilia in these heterozygotes is ultimately attributable to primary nonrandom $\mathrm{X}$ inactivation occurring during embryogenesis, at the onset of the inactivation process.

\section{References}

1 Seeler RA, Vnencak-Jones CL, Bassett LM, Gilbert JB, Michaelis RC: Severe haemophilia A in a female: a compound heterozygote with nonrandom X-inactivation. Haemophilia 1999; 5: 445-449.

2 David D, Morais S, Ventura C, Campos M: Female haemophiliac homozygous for the factor VIII intron 22 inversion mutation, with transcriptional inactivation of one of the factor VIII alleles. Haemophilia 2003; 9: 125-130.

3 Mazurier C, Parquet-Gernez A, Gaucher C, Lavergne JM, Goudemand J: Factor VIII deficiency not induced by FVIII gene mutation in a female first cousin of two brothers with haemophilia A. Br J Haematol 2002; 119: 390-392.

4 Migeon BR, McGinniss MJ, Antonarakis SE et al: Severe hemophilia $\mathrm{A}$ in a female by cryptic translocation: order and orientation of factor VIII within Xq28. Genomics 1993; 16: 20-25.

5 Valleix S, Vinciguerra C, Lavergne JM, Leuer M, Delpech M, Negrier C: Skewed X-chromosome inactivation in monochorionic diamniotic twin sisters results in severe and mild hemophilia A. Blood 2002; 100: 3034-3036.

6 Nance WE: Do twin Lyons have larger spots? Am J Hum Genet 1990; 46: 646-648.

7 Nisen PD, Waber PG: Nonrandom X chromosome DNA methylation patterns in hemophiliac females. J Clin Invest 1989; 83: $1400-1403$.

8 Acquila M, Caprino D, Bicocchi P, Mori PG, Tagliaferri AR: A skewed lyonization phenomenon as cause of hemophilia A in a female patient. Blood 1995; 85: 599-600.

9 Favier R, Lavergne JM, Costa JM et al: Unbalanced X-chromosome inactivation with a novel FVIII gene mutation resulting in severe hemophilia A in a female. Blood 2000; 96: 4373-4375.

10 Coleman R, Genet SA, Harper JI, Wilkie AO: Interaction of incontinentia pigmenti and factor VIII mutations in a female with biased X inactivation, resulting in haemophilia. J Med Genet 1993; 30: 497-500.

11 Sharp A, Robinson D, Jacobs P: Age- and tissue-specific variation of $\mathrm{X}$ chromosome inactivation ratios in normal women. Hum Genet 2000; 107: 343-349.

12 Puck JM, Willard HF: X inactivation in females with X-linked disease. N Engl J Med 1998; 338: 325-328. 
13 Migeon BR: Non-random X chromosome inactivation in mammalian cells. Cytogenet Cell Genet 1998; 80: 142-148.

14 Raynaud M, Moizard MP, Dessay B et al: Systematic analysis of $\mathrm{X}$-inactivation in 19XLMR families: extremely skewed profiles in carriers in three families. Eur J Hum Genet 2000; 8: 253-258.

15 Avner P, Heard E: X chromosome inactivation: counting, choice and initiation. Nat Rev Genet 2001; 2: 59-67.

16 Percec I, Plenge RM, Nadeau JH, Bartolomei MS, Willard HF: Autosomal dominant mutations affecting $\mathrm{X}$ inactivation choice in the mouse. Science 2002; 296: 1136-1139.

17 Brown CJ, Hendrich BD, Rupert JL et al: The human XIST gene: analysis of a $17 \mathrm{~kb}$ inactive X-specific RNA that contains conserved repeats and is highly localized within the nucleus. Cell 1992; 71: 527-542.

18 Hendrich BD, Brown CJ, Willard HF: Evolutionary conservation of possible functional domains of the human and murine XIST genes. Hum Mol Genet 1993; 2: 663-672.

19 Oldenburg J, Ivaskevicius V, Rost S et al: Evaluation of DHPLC in the analysis of hemophilia A. J Biochem Biophys Methods 2001; 47: $39-51$.

20 Bicocchi MP, Pasino M, Lanza $\mathrm{T}$ et al: Analysis of 18 novel mutations in the factor VIII gene. Br J Haematol 2003; 122: $810-817$.
21 Plenge RM, Hendrich BD, Schwartz C et al: A promoter mutation in the XIST gene in two unrelated families with skewed X-chromosome inactivation. Nat Genet 1997; 17: 353-356.

22 Allen RC, Zoghbi HY, Moseley AB, Rosenblatt HM, Belmont JW: Methylation of HpaII and HhaI sites near the polymorphic CAG repeat in the human androgen-receptor gene correlates with $\mathrm{X}$ chromosome inactivation. Am J Hum Genet 1992; 51: 1229-1239.

23 Rupert JL, Brown CJ, Willard HF: Direct detection of non-random $\mathrm{X}$ chromosome inactivation by use of a transcribed polymorphism in the XIST gene. Eur J Hum Genet 1995; 3: 333-343.

24 Kemball-Cook G, Tuddenham EG, Wacey AI: The factor VIII structure and mutation resource site: HAMSTeRS version 4 . Nucleic Acids Res 1998; 26: 216-219.

25 Pereira LV, Zatz M: Screening of the C43G mutation in the promoter region of the XIST gene in females with highly skewed X-chromosome inactivation. Am J Med Genet 1999; 87: $86-87$.

26 Naumova AK, Plenge RM, Bird LM et al: Heritability of $\mathrm{X}$ chromosome-inactivation phenotype in a large family. Am J Hum Genet 1996; 58: 1111-1119.

27 Naumova AK, Olien L, Bird LM et al: Genetic mapping of X-linked loci involved in skewing of $\mathrm{X}$ chromosome inactivation in the human. Eur J Hum Genet 1998; 6: 552-562. 